\title{
The role of improved social support for healthy eating in a lifestyle intervention: Texercise Select
}

\author{
Aya Yoshikawa $^{1, *}$ (1), Matthew Lee Smith ${ }^{1,2,3}$ (1), Shinduk Lee ${ }^{1}$ (1), Samuel D Towne Jr ${ }^{1,2,4,5,6}$ (1) \\ and Marcia G Ory ${ }^{1,2}$ (1) \\ ${ }^{1}$ Center for Population Health and Aging, School of Public Health, Texas A\&M University, 212 Adriance Lab Road, \\ 1266 TAMU, Suite 360, College Station, TX 77843-1266, USA: ${ }^{2}$ Department of Environmental and Occupational \\ Health, School of Public Health, Texas A\&M University, College Station, TX, USA: ${ }^{3}$ Deptartment of Health Promotion \\ and Behavior, College of Public Health, The University of Georgia, Athens, GA, USA: ${ }^{4}$ Department of Health \\ Management \& Informatics, College of Community Innovation and Education, University of Central Florida, Orlando, \\ FL, USA: ${ }^{5}$ Disability, Aging \& Technology Faculty Cluster Initiative, University of Central Florida, Orlando, FL, USA: \\ ${ }^{6}$ Southwest Rural Health Research Center, School of Public Health, Texas A\&M University, College Station, TX, USA
}

Submitted 19 December 2019: Final revision received 24 June 2020: Accepted 6 July 2020: First published online 24 August 2020

\begin{abstract}
Objective: We examined the measurement and mediating role of social support in dietary intake among participants in Texercise Select, an intervention for improving lifestyle behaviours.

Design: Quasi-experimental study. Participants reported their dietary intake, level of social support measured by the new Social Support for Healthy Eating scale, sociodemographics and disease profile. We conducted exploratory factor analysis for scale evaluation and structural equation modelling for mediation analysis to test if changes in dietary-specific social support mediate the relationship between the intervention and changes in dietary intake.

Setting: Texas.

Participants: Community-dwelling middle-aged and older adults completed a self-reported survey at baseline and 3-month follow-up (intervention group $n$ 211, comparison group $n$ 175).

Results: The majority of the sample was aged $\geq 70$ years (mean 74.30, SD 8.54), female $(82 \cdot 1 \%)$ and had at least two chronic conditions $(63.5 \%)$. The acceptable levels of reliability and validity of the dietary-specific social support scale were confirmed. Compared with the comparison group, the intervention group reported improved intake of fruit/vegetables and water, and improved dietary-specific social support. Improved dietary-specific social support mediated the association between intervention and change in fruit/vegetable intake, controlling for sociodemographics, number of chronic conditions and geographic residence. About $12 \%$ of intervention effect was mediated by social support.

Conclusions: The current study confirms positive intervention effects on healthy eating, and highlights social support relating to dietary behaviours that may be helpful for healthy eating. Future research should investigate additional social support for developing healthy eating behavioural skills.
\end{abstract}

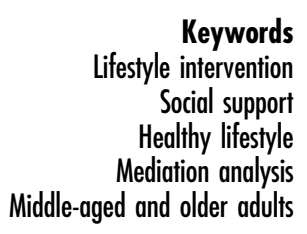

Identifying healthy lifestyles such as proper nutrition is critical to lessen the burden of chronic disease and related risk factors. Lifestyle interventions among middle-aged and older adults may be complex and may have differing effects based on social support. Thus, an investigation of determinants that may lead to one's healthy lifestyle is important. For example, lifestyle factors play a substantial role in the prevention and management of chronic diseases such as
CVD, obesity and type 2 diabetes, which are prevalent at substantial rates in middle-aged and older adults ${ }^{(1-3)}$. About $20 \%$ of adults aged $\geq 65$ years die of CVD, and one-third of deaths relating to CVD occur among those under 75 years of age ${ }^{(1)}$. According to a 2019 report, the United States spent approximately US $\$ 351$ billion on the medical costs of CVD and stroke in 2014-15, and the cost is projected to increase for middle-aged (45-64 years) 
and older ( $\geq 65$ years) adults in the future ${ }^{(1)}$. Furthermore, the growing prevalence of obesity among older adults is prominent; more than one-third of adults aged $\geq 60$ years (37.5\% for men and $39.4 \%$ for women) are obese ${ }^{(3)}$, increasing the risk of type 2 diabetes $^{(4)}$. Thus, multimorbidity among middle-aged and older populations has increasingly become a public health concern.

For the prevention and management of chronic disease, the importance of healthy eating habits, in addition to regular physical activity, has been emphasised ${ }^{(5)}$. Certain dietary patterns, such as the Mediterranean and plant-based diets, for example, are associated with reduced risk for $\mathrm{CVD}^{(6,7)}$, cognitive decline ${ }^{(8-10)}$, type 2 diabetes $^{(11-13)}$ and depression $^{(14)}$, as well as enhanced quality of life ${ }^{(15)}$. However, the intake of fruit/vegetables among older adults is suboptimal $^{(16)}$. Only $10 \cdot 9-12 \cdot 4 \%$ of adults aged $\geq 51$ years consume the recommended amount of fruit and vegetables to meet federal recommendations ${ }^{(17)}$. To reduce the risk of developing and accelerating chronic diseases, additional attention should be paid to factors that facilitate healthy dietary habits.

As a critical factor relating to health behaviour, research shows the influence of social relationships on dietary intake. Social isolation has been reported to increase the risk of poor nutrition ${ }^{(18-21)}$, while social support may help support healthy dietary behaviour of older adults ${ }^{(21-24)}$. For instance, an analysis of adults aged $\geq 50$ years in the European Prospective Investigation of Cancer (EPIC) study identified an association between being widowed or living alone and low levels of vegetable intake, and this relationship was more profound among men compared with women ${ }^{(21)}$. Those with less frequent contact with friends had lower fruit/vegetable intake. Similarly, the association between poor diet intake and living alone was reported in a study of the National Health and Nutrition Examination Survey III data. ${ }^{(25)}$ A focus group study of communitydwelling older adults in the UK also pointed out the positive influence of social engagement (e.g. social activity and social interaction with friends and family) on dietary habit ${ }^{(26)}$. A study of community-dwelling older adults in the $\mathrm{UK}^{(24)}$ found that men and women who had emotional support had a better diet, which was measured by a FFQ assessing the consumption of twenty-four foods like fruit, vegetables, wholegrain cereals and oily fish. Practical support was associated with healthy eating among older men, while a larger social network was more critical among older women ${ }^{(24)}$. Findings of these previous studies are mostly based on cross-sectional analysis ${ }^{(23)}$. Additionally, the concept of social support for a healthy diet has been predominantly captured by indirect measures, such as the number of friends; friendship density ${ }^{(22)}$; frequency of social $^{(20)}$, family and friend ${ }^{(21)}$ contact; and the availability of emotional, practical and negative aspects of support in general $^{(24,27)}$. Limited studies have assessed social support directly relating to dietary intake, such as companionship - having meals together (e.g. refs. 19 and 22) and helping with cooking ${ }^{(22)}$. The role of social support specific to healthy eating among older adults has been understudied.

\section{Intervention}

To help lessen extant research gaps, the current study examined the role of social support on dietary intake among older adults who participated in a lifestyle intervention designed to improve eating habits and physical activity, Texercise Select ${ }^{(28)}$. Texercise Select is a 10-week group-based programme designed for adults aged $\geq 45$ years. The programme was designed to encourage healthy eating and physical activity through education, discussions and training exercise ${ }^{(28)}$. Led by trained facilitators who go through $6 \mathrm{~h}$ of standardised training, this programme uses an official programme manual and other complementary materials. The programme delivers a total of twenty sessions (i.e. two $1.5-\mathrm{h}$ sessions each week for 10 weeks), and about a half of the workshops were devoted to discussions and activities about healthy eating. In each session, programme participants discuss healthy eating and behaviour changes, such as goal-setting for healthy eating, nutrition facts, how to read food labels, behaviour tracking and problem-solving in a group setting.

The programme was designed to help participants gain different behavioural skills through hands-on practice and discussions about how to overcome barriers and meet goals associated with healthy eating. The effectiveness of Texercise Select has been reported: improved fruit/ vegetable intake per week ${ }^{(29,30)}$, fast-food intake per week $^{(30)}$, daily water intake ${ }^{(29,30)}$, physical activity ${ }^{(29,31)}$ and sedentary behaviour ${ }^{(31)}$. Moreover, programme participants improved the level of social support for physical $\operatorname{activity}^{(31)}$ and dietary intake ${ }^{(29,30)}$.

\section{Aims}

Given the relationship between dietary-specific social support and food intake has not been fully explored, the aims of the present study were to: (i) evaluate the psychometrics of the dietary-specific social support scale included in the current study, the Social Support for Healthy Eating Scale; and (ii) test if improved dietary-specific social support mediates the intervention effect on changes in dietary intake among Texercise Select participants.

\section{Methods}

\section{Participants and study procedure}

Data were collected from middle-aged and older adults who resided in Texas and who were recruited from various community sites between May 2015 and Aug 2017. Participants were recruited through various communitybased organisations for older adults and healthcare centres 
that provide health and wellness programmes to older adults in Texas ${ }^{(31)}$. While the research team approached and recruited participants who have similar characteristics, the intervention and comparison groups were not randomly assigned due to the pragmatic nature of the communitybased intervention. Group assignment was determined based on the need and preference of participating sites. For the intervention group, Texercise Select workshops were delivered in community settings such as senior centres, senior housing, community centres and faith-based facilities. Texercise Select was not delivered to the comparison group, although the participants in this group might have been exposed to other health and wellness programmes. The current study used data from 386 middle-aged and older adults (intervention $n 211$, comparison $n$ 175) who completed pre- and post-surveys.

\section{Dietary intake}

Information about dietary intake was collected using four items $^{(29)}$. Participants were asked to answer the question, 'Over the past 7 days, how many times did you eat fast food, meals or snacks?' using a six-point scale: '0', '1', '2', '3', '4' and ' $\geq 5$ '. Using the same response scale, the following three questions were asked: 'Over the past 7 days, how many servings of fruit/vegetables did you eat each day?' and 'Over the past 7 days, how many soda and sugarsweetened drinks (regular, not diet) did you drink each day?' Daily water intake was assessed with the item 'In the average day, how many cups of water do you drink each day?' using a nine-point scale, ranging from ' 0 ' to ' $\geq 8$ '. Change scores between pre- and post-survey values were derived by regressing post-survey values on pre-survey values.

\section{Dietary-specific social support}

The current study used an original Social Support for Healthy Eating Scale to assess changes in dietary-specific social support. Participants rated how often they receive social support by assessing the following three specific social support goals for dietary activities: 'plan dietary goals', 'keep dietary goals' and 'reduce barriers to healthy eating'. The perceived availability of dietary-specific social support was reported using a four-point scale with categories of 'never', 'rarely', 'sometimes' and 'often'. Similarly, we computed the change scores between pre- and post-survey values by regressing post-survey values on pre-survey values. In Texercise Select, participants discuss how to plan and keep goals as well as remove barriers to healthy eating in a group setting. This scale consisting of three items relating to workshop discussion topics was used to evaluate programme effectiveness - whether participants improved levels of dietary-specific social support as a result of Texercise Select workshop participation. While having reported the internal consistency of the scale (i.e. Cronbach's $\alpha$ ) in the previous studies ${ }^{(29,31)}$, this is the first study that investigated the reliability and validity of the scale.

\section{Sociodemographic information}

Participants' sociodemographic information collected by the questionnaire included age, sex, living arrangement, race, chronic conditions and education. Age (number of years), sex (female $=1$, male $=0$ ), race (white $=1$, nonwhite $=0)$, education $(0=$ less than some high school, $1=$ some high school, 2 = high school graduate, $3=$ some college or vocational school, $4=$ college graduate or higher), number of chronic conditions (self-reported), living arrangement (living alone $=1$, living with someone $=0$ ) were entered as covariates in the mediation analysis.

Participants selected their race from the categories of White, Black or African American, Asian, American Indian/Alaska Native, Native Hawaiian or Pacific Islander, and other. Another item assessed their ethnicity (Hispanic/ Latino $=1$, non-Hispanic/Latino $=0$ ). Because a vast majority (90.0 \%) of participants reported being non-Hispanic/Latino, we elected to use race (and omit ethnicity) from study analyses to facilitate meaningful comparisons. Since a vast majority of our analytic sample were either White (50.0\%) or Black or African American (46.5\%) (i.e. $3.6 \%$ for Asian or other), a binary race category (i.e. White or Non-white, which includes Black or African American, Asian, American Indian/ Alaska Native, Native Hawaiian or Pacific Islander and other) was created and used for analyses.

Participants were provided a list of chronic conditions and asked to self-report that they had been diagnosed with by a healthcare provider. The list included arthritis/ rheumatic disease, breathing/lung disease (e.g. asthma, emphysema and bronchitis), cancer, depression or anxiety disorder, diabetes, heart disease, hypertension and stroke. The number of chronic conditions was derived from the sum of endorsed conditions from this selfreported list.

Food access was reported as a factor relating to dietary intake among older adults ${ }^{(32)}$; therefore, we also included geographic residence as a covariate using the 2013 National Center for Health Statistics' Urban-Rural Classification Scheme for the Counties of Centers for Disease Control and Prevention ${ }^{(33)}$. This coding scheme consists of six levels based on: (i) metropolitan statistical areas (MSA) and micropolitan statistical areas defined by the Office of Management and Budget, (ii) the population size of MSA and (iii) the location of city populations in the largest MSA with $\geq 1$ million $^{(33)}$. In the current study, we created two geographical groups: large/medium metro (codes 1-3) and small metro/micropolitan/non-core (codes 4-6) counties. Based on this coding scheme, each participant's residence by county level was coded as ' 1 ' for large/medium metro and '0' for small metro/micropolitan/ non-core. 


\section{Data analysis}

\section{Basic between-group comparisons}

Sociodemographic characteristics and chronic conditions were compared between intervention and comparison groups using a $\chi^{2}$ test for categorical variables and a Mann-Whitney $U$ test for skewed continuous or ordinal variables.

\section{Attrition analyses}

We performed an attrition analysis to assess whether there were significant differences in sociodemographic characteristics between the participants who completed the pre- and post-surveys after the programme and those who did not.

\section{Scale evaluation}

We performed an exploratory factor analysis to examine whether the three items of the dietary-specific social support measured the same construct using data from the study participants who completed the pre-survey ( $n$ 564). In addition to bivariate correlations for the identification of possible multicollinearity, sampling adequacy (Kaiser-Meyer-Olkin [KMO] value $>0.5$ ) and Bartlett's test for patterned relationships $(P<0.05)^{(34)}$ were assessed. We then examined the reliability and validity of the dietaryspecific social support construct - Social Support for Healthy Eating Scale - using the criteria: Cronbach's $\alpha>0.7$, composite reliability score $>0.7$ and average variance extracted (i.e. convergent validity) $>0 \cdot 5^{(35)}$.

\section{Total intervention effect}

We assessed associations between the intervention condition ( $1=$ intervention, $0=$ comparison $)$ and changes in dietary intake, controlling for age, sex, race, number of chronic conditions, living arrangement and geographic residence.

\section{Mediational analysis}

The current study employed structural equation modelling to test if changes in dietary-specific social support mediated the relationship between the intervention condition and changes in dietary intake, controlling for age, sex, race, number of chronic conditions, living arrangement and geographic residence (Fig. 1). For estimating changes in outcome variables and the potential mediator (i.e. dietaryspecific social support) over the intervention period, we computed the residualised change scores by regressing post-survey scores on the corresponding pre-survey values $^{(36)}$. Maximum likelihood with robust standard error method was used for structural equation modelling since the endogenous variables (i.e. residualised change scores) were continuous and assumed to have non-normal distributions. For mediation testing, we used the biascorrected bootstrapping method, which has an advantage over the conventional mediation testing method ${ }^{(37)}$ for constructing confidence intervals of parameters regardless of

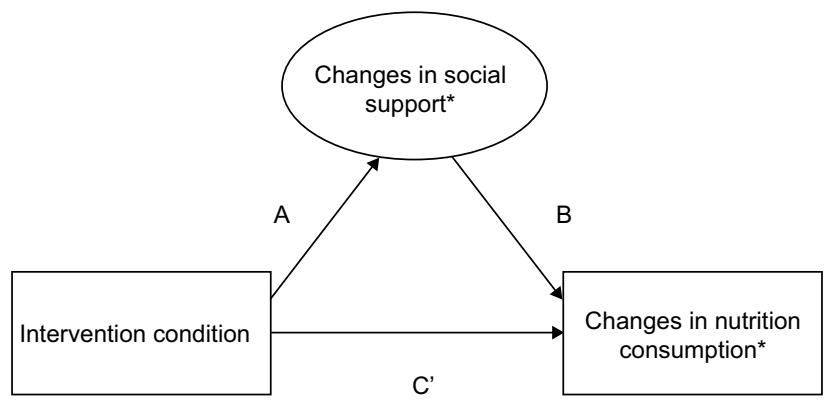

Fig. 1 The model depicting the mediating role of social support between intervention condition and changes in dietary intake. ${ }^{*}$ Residual change scores obtained based on pre-survey scores as predictors

the assumption of normal distribution ${ }^{(38)}$. Data were missing completely at random (MCAR) (Little's MCAR test $=331.45, P=0.192$ ); thus, we used the Full Information Maximum Likelihood estimation method for mediation testing. Exploratory factor analysis and structural equation modelling were performed using SPSS 25 (IBM SPSS Statistics) and Mplus 8 (Muthen \& Muthen), respectively.

\section{Results}

A majority of participants in the intervention and comparison groups were female $(81.9 \%$ for intervention, $76.8 \%$ for comparison), $\geq 75$ years ( $46.2 \%$ for intervention, $51.3 \%$ for comparison), had at least some college or vocation school education (63.6 \% for intervention, 58.9\% for comparison) and had $\geq 2$ chronic conditions (64.8\% for intervention, $57.9 \%$ for comparison) (Table 1). About half of older adults lived with someone $(52.2 \%$ for intervention, $50.6 \%$ for comparison). The intervention group had a significantly larger proportion of White participants $\left(\chi^{2}=7 \cdot 00\right.$, $\mathrm{df}=1, P=0.008)$ and large/medium metro county residence $\left(\chi^{2}=26.89\right.$, df $\left.=1, P<0.001\right)$ than the comparison group. No significant difference at pre-survey was found in dietary intake between the intervention and comparison groups. No other items in dietary-specific social support differed between intervention and comparison groups.

Supplementary analyses showed that among retention and attrition groups, there were no significant differences in sociodemographic, dietary intake or dietary-specific social support, except social support for reducing barriers to healthy eating at pre-survey for the intervention group ( $z=-0.21, P=0.044$ ) (Table 2). Among the intervention group, a higher level of social support for reducing barriers to healthy eating was observed in those who completed both pre- and post-surveys.

\section{Scale evaluation}

The acceptable reliability and validity of the dietary-specific social support scale (Social Support for Healthy Eating) 
Table 1 Sociodemographic and health characteristics, dietary intake and social support of participants at pre-survey by intervention and comparison groups

\begin{tabular}{|c|c|c|c|c|c|}
\hline & \multicolumn{2}{|c|}{$\begin{array}{l}\text { Intervention } \\
\text { (n 293) }\end{array}$} & \multicolumn{2}{|c|}{$\begin{array}{c}\text { Comparison } \\
(n 271)\end{array}$} & \multirow[b]{2}{*}{ Group test, $F$} \\
\hline & $n$ & $\%$ & $n$ & $\%$ & \\
\hline \multicolumn{6}{|l|}{ Sex } \\
\hline Female ( \%) & 240 & 81.9 & 208 & $76 \cdot 8$ & 0.159 \\
\hline \multicolumn{6}{|l|}{ Age } \\
\hline$<65$ years & 35 & $12 \cdot 1$ & 40 & $14 \cdot 8$ & $0 \cdot 155$ \\
\hline $65-74$ years & 121 & $41 \cdot 7$ & 92 & 33.9 & \\
\hline$\geq 75$ years & 134 & $46 \cdot 2$ & 139 & $51 \cdot 3$ & \\
\hline Race & & & & & 0.008 \\
\hline White & 158 & 53.9 & 115 & $42 \cdot 4$ & \\
\hline Non-White* & 135 & $46 \cdot 1$ & 156 & $57 \cdot 6$ & \\
\hline Living arrangement & & & & & 0.757 \\
\hline Living alone & 140 & $47 \cdot 8$ & 113 & $49 \cdot 4$ & \\
\hline Geographic residence & & & & & $<0.001$ \\
\hline Large/medium metro & 272 & $92 \cdot 8$ & 207 & $77 \cdot 0$ & \\
\hline Small metro/micropolitan/non-core & 21 & $7 \cdot 2$ & 62 & 23.0 & \\
\hline \multicolumn{6}{|l|}{ Education } \\
\hline Less than some high school & 22 & $7 \cdot 6$ & 28 & $10 \cdot 4$ & 0.085 \\
\hline Some high school & 22 & $7 \cdot 6$ & 24 & 8.9 & \\
\hline High school graduate & 62 & $21 \cdot 3$ & 59 & 21.9 & \\
\hline Some college or vocation school & 112 & 38.5 & 75 & $27 \cdot 8$ & \\
\hline College graduate or higher & 73 & $25 \cdot 1$ & 84 & $31 \cdot 1$ & \\
\hline Number of chronic conditions (0-8) & & & & & 0.132 \\
\hline None & 35 & 11.9 & 47 & $17 \cdot 3$ & \\
\hline 1 & 68 & $23 \cdot 2$ & 67 & $24 \cdot 7$ & \\
\hline \multirow[t]{2}{*}{$\geq 2$} & 190 & $64 \cdot 8$ & 157 & $57 \cdot 9$ & \\
\hline & Mean & SD & Mean & SD & $P$ \\
\hline \multicolumn{6}{|l|}{ Dietary intake } \\
\hline Number of fast-food meals or snacks the person ate in the past $7 \mathrm{~d} \dagger$ & 1.88 & 1.58 & 1.91 & 1.58 & 0.698 \\
\hline Number of servings of fruit/vegetables the person ate each day in the past $7 \mathrm{~d} \dagger$ & 3.33 & 1.42 & $3 \cdot 16$ & 1.30 & 0.319 \\
\hline Number of soda and sugar-sweetened drinks the person had each day in the past $7 \mathrm{~d} \dagger$ & 1.04 & 1.29 & $1 \cdot 15$ & 1.49 & 0.382 \\
\hline Cups of water the person drinks each day on an average day $\ddagger$ & $5 \cdot 22$ & 2.07 & 5.04 & $2 \cdot 19$ & 0.556 \\
\hline \multicolumn{6}{|l|}{ Social support§ } \\
\hline Plan dietary goals & $2 \cdot 36$ & $1 \cdot 21$ & $2 \cdot 32$ & 1.24 & 0.715 \\
\hline Keep dietary goals & $2 \cdot 46$ & $1 \cdot 18$ & $2 \cdot 32$ & $1 \cdot 21$ & 0.736 \\
\hline Reduce barriers to healthy eating & $2 \cdot 47$ & $1 \cdot 26$ & 2.35 & 1.21 & 0.426 \\
\hline
\end{tabular}

*Including Black or African American, Asian, American Indian/Alaska Native, Native Hawaiian or Pacific Islander and other.

†Six-point scale $(0,1,2,3,4, \geq 5)$.

¥Nine-point scale $(0,1,2,3,4,5,6,7, \geq 8)$.

§Four-point scale ( $1=$ never, 2 = rarely, $3=$ sometimes, $4=$ often).

was confirmed. Correlations among the three items of dietary-specific social support were moderate to strong $(0.57-0.75)$. No violation of multicollinearity for the construct was confirmed (determinant value 0.23). The obtained KMO value (0.69) indicated an adequate level for factor analysis ${ }^{(39)}$. Patterned relationships were also observed among the three items of dietary-specific social support (Bartlett's test of sphericity: $\chi^{2}=813 \cdot 10, \mathrm{df}=3$, $P<0.001)$. The constructs of dietary-specific social support showed high internal consistency with Cronbach's $\alpha(0 \cdot 86)$. The obtained values of composite reliability (0.86) and average variance extracted (i.e. convergent validity) (0.68) met the $\operatorname{criteria}^{(35)}$.

\section{Total intervention effects}

After the intervention period, the intake of fruit/vegetables $\left(B_{c}=0.52, \mathrm{SE}=0.13, \quad P<0.001\right)$ and water $\left(B_{c}=0.31\right.$, $\mathrm{SE}=0 \cdot 15, P=0.041)$ increased in the intervention group compared with the comparison group, controlling for sex, age, race, education, number of chronic conditions, living arrangement and geographic residence (direct effect) (Table 3). Moreover, living with someone $(B=-0 \cdot 11$, $\mathrm{SE}=0.03, P=0.002)$ was associated with changes in fruit/ vegetable intake. No significant change was found in the intake of fast-food meals or snacks and soda/sugar-sweetened drinks in the intervention group compared with the comparison group $(P>0.05)$.

\section{Intervention effects on dietary-specific social support for practicing bealthy eating}

Participants reported significantly higher levels of dietaryspecific social support $\left(B_{A}=0 \cdot 28, \mathrm{SE}=0 \cdot 10, P=0 \cdot 003\right)$ in the intervention group compared with the comparison group after adjusting for sex, age, race, education, number of chronic conditions, living arrangement and geographic residence (direct effect) (Table 3). None of the included 
Table 2 Sociodemographic and health characteristics, dietary intake and social support of attrition and retention by group

\begin{tabular}{|c|c|c|c|c|c|c|c|c|c|c|}
\hline & \multicolumn{5}{|c|}{ Intervention } & \multicolumn{5}{|c|}{ Comparison } \\
\hline & \multicolumn{2}{|c|}{$\begin{array}{l}\text { Retention } \\
\quad(n 211)\end{array}$} & \multicolumn{2}{|c|}{$\begin{array}{l}\text { Attrition } \\
(n 82)\end{array}$} & \multirow{2}{*}{$\begin{array}{l}\text { Group } \\
\text { test, } P\end{array}$} & \multicolumn{2}{|c|}{$\begin{array}{l}\text { Retention } \\
(n \text { 175) }\end{array}$} & \multicolumn{2}{|c|}{$\begin{array}{l}\text { Attrition } \\
(n 96)\end{array}$} & \multirow{2}{*}{$\begin{array}{l}\text { Group } \\
\text { test, } F\end{array}$} \\
\hline & $n$ & $\%$ & $n$ & $\%$ & & $n$ & $\%$ & $n$ & $\%$ & \\
\hline \multicolumn{11}{|l|}{ Sex } \\
\hline Female (\%) & 178 & $84 \cdot 4$ & 62 & $75 \cdot 6$ & 0.115 & 139 & $79 \cdot 4$ & 69 & 71.9 & 0.209 \\
\hline \multicolumn{11}{|l|}{ Age } \\
\hline$<65$ years & 22 & $10 \cdot 5$ & 13 & $16 \cdot 0$ & 0.384 & 23 & $13 \cdot 1$ & 17 & $17 \cdot 7$ & 0.593 \\
\hline $65-74$ years & 87 & 41.6 & 34 & $42 \cdot 0$ & & 61 & 34.9 & 31 & $32 \cdot 3$ & \\
\hline$\geq 75$ years & 100 & $47 \cdot 8$ & 34 & $42 \cdot 0$ & & 91 & $52 \cdot 0$ & 48 & $50 \cdot 0$ & \\
\hline \multicolumn{11}{|l|}{ Race } \\
\hline White & 105 & $50 \cdot 2$ & 51 & $62 \cdot 2$ & 0.057 & 79 & $45 \cdot 1$ & 36 & 37.5 & 0.276 \\
\hline Non-White* & 106 & $49 \cdot 8$ & 31 & $37 \cdot 8$ & & 96 & 54.9 & 60 & 62.5 & \\
\hline \multicolumn{11}{|l|}{ Living arrangement } \\
\hline Living alone & 106 & $50 \cdot 2$ & 34 & 41.5 & 0.223 & 92 & 53.2 & 41 & $42 \cdot 7$ & 0.129 \\
\hline \multicolumn{11}{|l|}{ Geographic residence } \\
\hline Large/medium metro & 195 & 92.4 & 77 & 93.9 & 0.849 & 133 & $76 \cdot 9$ & 74 & $77 \cdot 1$ & 1.000 \\
\hline Small metro/micropolitan/noncore & 16 & $7 \cdot 6$ & 5 & $6 \cdot 1$ & & 40 & 23.1 & 22 & $22 \cdot 9$ & \\
\hline \multicolumn{11}{|l|}{ Education } \\
\hline Less than some high school & 15 & $7 \cdot 1$ & 7 & $8 \cdot 6$ & 0.521 & 16 & $9 \cdot 2$ & 12 & $12 \cdot 5$ & 0.242 \\
\hline Some high school & 15 & $7 \cdot 1$ & 7 & 8.6 & & 14 & 8.0 & 10 & $10 \cdot 4$ & \\
\hline High school graduate & 50 & $23 \cdot 8$ & 12 & $14 \cdot 8$ & & 44 & $25 \cdot 3$ & 15 & $15 \cdot 6$ & \\
\hline Some college or vocation school & 77 & $36 \cdot 7$ & 35 & 43.2 & & 51 & $29 \cdot 3$ & 24 & $25 \cdot 0$ & \\
\hline College graduate or higher & 53 & $25 \cdot 2$ & 20 & 24.7 & & 49 & $28 \cdot 2$ & 35 & $36 \cdot 5$ & \\
\hline Number of chronic conditions (0-8) & & & & & 0.814 & & & & & 0.308 \\
\hline 0 & 25 & 11.8 & 10 & $12 \cdot 2$ & & 26 & 14.9 & 21 & 21.9 & \\
\hline 1 & 47 & $22 \cdot 3$ & 21 & $25 \cdot 6$ & & 43 & $24 \cdot 6$ & 24 & $25 \cdot 0$ & \\
\hline \multirow[t]{2}{*}{$\geq 2$} & 139 & 65.9 & 51 & $62 \cdot 2$ & & 106 & $60 \cdot 6$ & 51 & 53.1 & \\
\hline & Mean & SD & Mean & SD & $P$ & Mean & SD & Mean & SD & $P$ \\
\hline \multicolumn{11}{|l|}{ Dietary intake } \\
\hline $\begin{array}{l}\text { Number of fast-food meals or snacks the person ate in the } \\
\text { past } 7 \mathrm{~d} \dagger\end{array}$ & $2 \cdot 06$ & 1.53 & $1 \cdot 81$ & 1.65 & 0.167 & 1.99 & 1.59 & 1.85 & 1.47 & 0.595 \\
\hline $\begin{array}{l}\text { Number of servings of fruit/vegetables the person ate each } \\
\text { day in the past } 7 \mathrm{~d} \dagger\end{array}$ & 3.34 & 1.39 & $3 \cdot 12$ & 1.43 & 0.277 & $3 \cdot 11$ & 1.37 & $3 \cdot 22$ & $1 \cdot 31$ & 0.628 \\
\hline $\begin{array}{l}\text { Number of soda and sugar-sweetened drinks the person } \\
\text { had each day in the past } 7 d(0-5) \dagger\end{array}$ & $1 \cdot 26$ & 1.60 & 0.90 & 1.34 & 0.105 & $1 \cdot 16$ & 1.41 & $1 \cdot 23$ & 1.50 & 0.725 \\
\hline $\begin{array}{l}\text { Cups of water the person drinks each day on an average } \\
\text { day }(0-8) \ddagger\end{array}$ & $5 \cdot 28$ & $2 \cdot 06$ & 5.05 & $2 \cdot 12$ & 0.446 & 5.09 & $2 \cdot 12$ & $5 \cdot 10$ & $2 \cdot 17$ & 0.860 \\
\hline \multicolumn{11}{|l|}{ Social support§ } \\
\hline Plan dietary goals & $2 \cdot 33$ & $1 \cdot 20$ & $2 \cdot 18$ & $1 \cdot 23$ & 0.394 & $2 \cdot 33$ & $1 \cdot 21$ & $2 \cdot 32$ & 1.22 & 0.960 \\
\hline Keep dietary goals & 2.48 & 1.15 & 2.37 & $1 \cdot 16$ & 0.492 & 2.46 & 1.18 & 2.27 & 1.20 & 0.225 \\
\hline Reduce barriers to healthy eating & 2.63 & 1.68 & $2 \cdot 27$ & $1 \cdot 24$ & $0.044 \|$ & 2.48 & 1.20 & 2.39 & 1.22 & 0.665 \\
\hline
\end{tabular}

*Including Black or African American, Asian, American Indian/Alaska Native, Native Hawaiian or Pacific Islander, and other.

†Six-point scale $(0,1,2,3,4, \geq 5)$.

¥Nine-point scale $(0,1,2,3,4,5,6,7, \geq 8)$.

$\S$ Four-point scale $(1=$ never, $2=$ rarely, $3=$ sometimes, $4=$ often $)$.

$\| P<0.05$.

covariates were associated with changes in the level of dietary-specific social support $(P>0.05)$.

\section{Influence of changes in dietary-specific social support on changes in dietary intake}

Changes in the level of dietary-specific social support after the intervention period were positively associated with changes in fruit/vegetable intake $\left(B_{B}=0.27, \mathrm{SE}=0.08\right.$, $P<0.001)$ after controlling for sex, age, race, education, number of chronic conditions, living arrangement and geographic residence (direct effect) (Table 3). Living with someone $(B=-0.11, \mathrm{SE}=0.03, P=0.002)$, female sex $(B=0.07, \mathrm{SE}=0.03, P=0.005)$ and large/medium metro residence $(B=0.06, \mathrm{SE}=0.03, P=0.023)$ were related to increases in fruit/vegetable intake. No association was found between changes in the level of dietary-specific social support and water intake $(P>0.05)$. Thus, only the model of fruit/vegetable intake was tested in the subsequent mediating analysis.

\section{Mediation analysis}

The mediating (indirect) effect of changes in the level of dietary-specific social support over the intervention period was confirmed on the relationship between intervention status and increased fruit/vegetable intake among programme participants, after controlling for sex, age, 
Table 3 Unstandardised path coefficients and significant tests (direct paths) from the structural equation modelling of intervention, social support and dietary intake†

\begin{tabular}{llrrrl}
\hline Causal path & & $\beta$ & SE & $P$ & Associated covariates* $^{*}$ \\
\hline Intervention & Fast-food meal or snack intake & -0.07 & 0.13 & 0.579 & \\
& Fruit/vegetable intake & 0.52 & 0.13 & $<0.001^{*}$ & Living with someone \\
& Soda and sugar-sweetened drink intake & -0.18 & 0.13 & 0.163 & Non-White race \\
& Water intake & 0.31 & 0.15 & $0.041^{*}$ & \\
& Levels of social support & 0.28 & 0.10 & $0.003^{*}$ & \\
Level of social support & -0.01 & 0.09 & 0.909 & Living with someone, female sex, \\
& Fast-food meal or snack intake & 0.27 & 0.08 & $<0.001^{*}$ & Large/medium metro residence \\
& Fruit/vegetable intake & & & & \\
& Soda and sugar-sweetened drink intake & 0.00 & 0.08 & 0.966 & Non-White race \\
& Water intake & -0.01 & 0.12 & 0.904 & \\
\hline
\end{tabular}

${ }^{*} P<0.05$.

†Each path controlled for age, sex, race, education, living arrangement, number of chronic conditions and geographic residence.

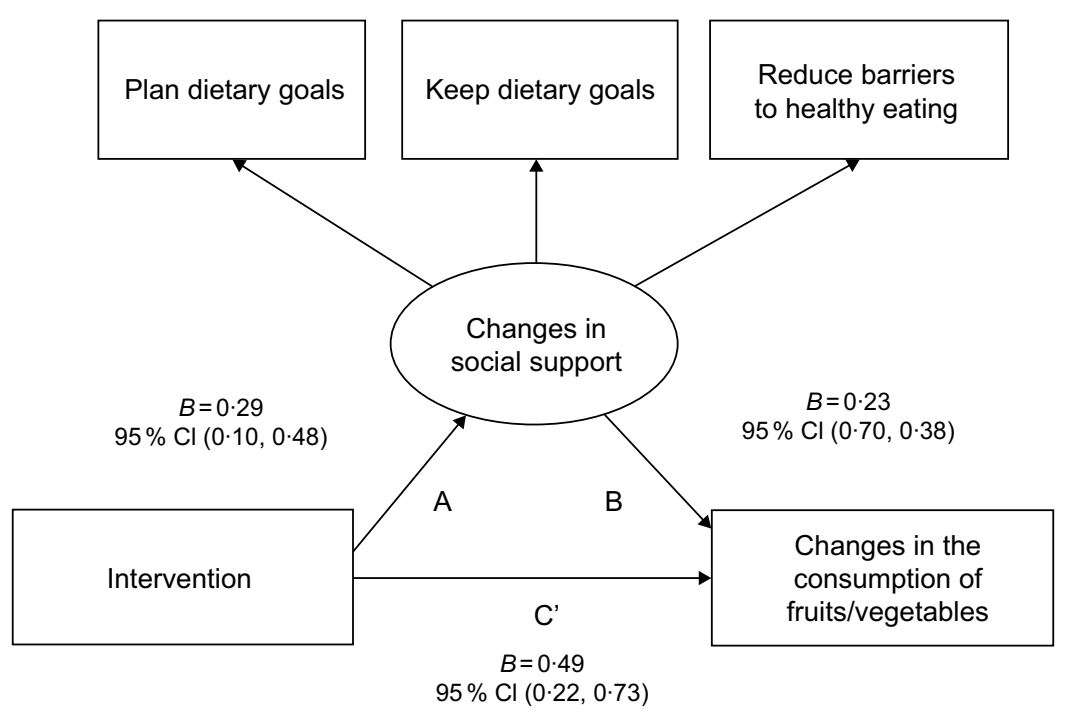

Fig. 2 Unstandardised coefficients and bias-corrected bootstrapped 95\% confidence intervals for direct and indirect effects from structural equation model testing the mediating (indirect) effect of changes in the level of social support between intervention and changes in fruit/vegetable intake. Note: The model controlled for age, sex, race, education, living arrangement, the number of chronic conditions and geographic residence

race, education, number of chronic conditions, living arrangement and geographic residence (Fig. 2). Changes in the level of dietary-specific social support mediated ( $\left.B_{A B}=0.06, \mathrm{SE}=0.02,95 \% \mathrm{CI} 0.01,0.15\right)$ the effects of the intervention on fruit/vegetable intake (indirect effect). Thus, the product $\left(B_{A B}\right)$ of direct effects fell outside of zero, indicating a significant indirect effect. The explained variance in the indirect effect of dietary-specific social support was $12.0 \%$. Living with someone $(B=-0 \cdot 10, \mathrm{SE}=0.04$, $P=0.003)$ and female sex $(B=0.07, \mathrm{sE}=0.03, P=0.010)$ were also associated with changes in fruit/vegetable intake. Regarding the dietary-specific social support items, keeping dietary goals $(\beta=0.93$, sE $=0.03, \quad P<0.001)$ made the strongest contribution in the mediation model, followed by planning dietary goals $(\beta=0.80, \mathrm{SE}=0.03$, $P<0.001)$ and reducing barriers to healthy eating $(\beta=0.70, \mathrm{sE}=0.04, P<0.001)$.

\section{Discussion}

The current study examined the mediating role of dietary-specific social support in dietary intake among the participants of Texercise Select, a lifestyle intervention. Confirming the appropriate psychometrics of our Social Support for Healthy Eating Scale, we found that improved dietary-specific social support mediated the intervention effect on improvement in fruit/vegetable intake over that intervention period. The indirect (mediation) effect accounted for about $12 \%$ of the overall intervention effect on the increase in fruit/vegetable intake. Moreover, compared with the comparison group, the current study also confirmed that programme participants improved the intake of fruit/vegetables and water as well as their level of dietary-specific social support. The improvement of dietary-specific social support among programme 
participants was associated with improved weekly fruit/ vegetable intake, but not with water intake. These findings suggest that middle-aged and older adults benefitted from this programme by improving fruit/vegetable intake that is partially enhanced by improved dietary-specific social support.

Moreover, we found that living arrangement, female sex and large/medium metro residence were associated with fruit/vegetable intake. Participants who lived with someone improved their fruit/vegetable intake regardless of improvement in dietary-specific social support. These findings suggest that middle-aged and older adults who live with someone may have had more opportunities to apply the healthy eating strategies that were discussed in Texercise workshops (i.e. goal-setting, behaviour tracking and problem-solving for potential barriers) despite the availability of perceived dietary-specific social support.

Previous studies reported consistent findings about the association between living arrangement and the risk of poor dietary intake among middle-aged and older adults $^{(25,32,40)}$. A secondary data analysis using the Nationwide Food Consumption Survey ${ }^{(40)}$ reported the risks of unhealthy eating behaviours among older adults who live alone, such as skipping meals (especially breakfast) and a higher proportion of calorie intake from eating outside of home. An association of living alone with a lower intake of fruit and vegetables was also confirmed in the study that examined the distance for a good selection (fresh or/and processed) of fruit or vegetables among older adults in rural areas ${ }^{(32)}$. Furthermore, a study of the National Health and Nutrition Examination Survey III data reported that living alone was associated with poor diet quality among middle-aged and older adults ${ }^{(40)}$.

We found a significant association of living with someone with improved fruit/vegetable intake in this programme evaluation. Our study examined the effect of lifestyle intervention by investigating changes in the dietary intake immediately before and at the end of intervention. The influences of living arrangement on healthy eating among older adults in general as well as in programme evaluation need to be further examined for promoting healthy eating.

We also found that being female was associated with improved fruit/vegetable intake in the mediation model. The findings imply that among female participants of Texercise Select, improvement in fruit/vegetable intake was partially due to improved dietary-specific social support during the workshops. Future studies should examine how female sex plays a role in improving social support for healthy eating. The differential associations of dietaryspecific social support with dietary intake by sex have been addressed $^{(18,24)}$. A larger social network was a factor associated with better diet quality among older women, while the availability of practical support was important among older men ${ }^{(24)}$. The lower level of perceived dietary-specific social support was also associated with the risk of poor nutrition among White older women (but not with Black women $)^{(18)}$. Although differences in dietary-specific social support by race and ethnicity need to be further investigated, the present and previous studies confirmed the differential influences of social relation or support on eating habits by sex.

The current study also identified an association between residing in a large/medium metro area and increased fruit/vegetable intake. This finding can be explained by the ease of access to food resources in large or medium metropolitan areas compared with less-dense small metropolitan or rural areas. A study of food access among older adults in rural areas ${ }^{(32)}$ reported that lower levels of fruit and vegetable intake were associated with a longer distance to the nearest supermarket and food stores that sold a variety of fruit and vegetables, after controlling for sociodemographic characteristics and perceived food access. However, in another study, distance to supermarkets in urban areas was not identified as a factor associated with fruit and vegetable intake ${ }^{(41)}$. Inadequate transportation is a known factor influencing limited food choices among rural older adults ${ }^{(42)}$. Thus, our study findings suggest that older adults who reside in large or medium metropolitan areas benefit from the availability of wider food selections, more grocery stores and public transportation. Workshop participants in urban areas may have had more opportunities to obtain healthy foods and apply healthy eating skills because they resided in communities with more resources.

The present study assessed the level of social support directly relating to healthy eating. Similar findings have documented positive associations of social support with dietary intake among older adults, yet they were predominantly based on general social support measures. More specifically, these studies used indirect support measures such as marital status ${ }^{(18)}$, social contact ${ }^{(20)}$, social engagement ${ }^{(43)}$, friendship network ${ }^{(22)}$, social support in general (i.e. instrumental support, emotional support, social interaction and social space) ${ }^{(44)}$ and adapted social support scales (e.g. the Arthritis Impact Measurement Scale for Social Support ${ }^{(45)}$ and the Close Persons Questionnaire $\left.{ }^{(27)}\right)$. Limited studies have assessed social support specific to dietary behaviours, such as companionship measured by spending meal time together ${ }^{(19,22)}$ and helping with cooking ${ }^{(22)}$. When assessing diet-related outcomes among participants of a lifestyle intervention that includes components specifically targeting a healthy diet, the use of social support scales directly assessing healthy eating behaviours is critical. For example, Texercise Select participants discuss specific healthy eating strategies, such as goal-setting, proper portion and food labels, in group-based workshops. The direct measure of social support for healthy eating can help identify specific social support associated with improved dietary intake. 


\section{Limitations}

Several limitations should be noted. First, the current study was conducted without randomisation because of the nature of interventions in community settings. Thus, the estimates of true intervention effects are limited; yet external validity was enhanced in this pragmatic research study ${ }^{(46)}$. That said, the current study employed a pre/post caseand-comparison design with an intervention group exposed to the intervention and a comparison group not exposed to the intervention. This is a significant strength beyond a simple pre-post case design. Second, the current study used data from a convenience sample, which may limit the sufficiency of sample size. To compensate for this potential limitation, we employed the bias-corrected bootstrapping method - a non-parametric method of testing indirect effects - in mediation analysis ${ }^{(38)}$. This statistical method allowed us to generate a pseudo-random sample, which may be small or moderate and have an asymmetrical distribution. Third, the geographic scope of the current study was limited (i.e. selected communities in Texas, limited sample distribution preventing a clear rural/urban distinction); therefore, the findings may not be generalisable to the larger population of Texas or that of the nation as a whole. It should also be noted that the focus was on community-dwelling middle-aged and older adults, and may not be generalisable to middle-aged and older adults residing in an institutionalised setting. Fourth, the current study utilised self-report information; thus, risk of errors, such as recall bias and social desirability bias, could not be avoided ${ }^{(47)}$. A recall error could influence the association between dietary-specific social support and dietary intake. It should be also noted that there is a possibility that self-report data could be influenced by one's social desirability or acceptability, despite the current study addressing anonymity and confidentiality during data collection.

A relatively large proportion of participants did not complete post-survey (28.0\% for intervention, $35.4 \%$ for comparison). To minimise potential bias due to attrition, we performed attrition analysis to investigate sociodemographic and health status differences. We adjusted the analyses for race, which significantly differed between completers of pre- and post-survey and noncompleters when performing structural equation modelling (in addition to age, sex, education, living arrangement, number of chronic conditions and geographic residence). Moreover, we had imbalanced data in the sex variable with a large proportion (80.0 \%) of female participants typical of community-based health promotion programmes. Thus, our findings about the association between being female and having increased fruit/vegetable intake may require a careful interpretation for generalisability. Given the distribution of this variable, we acknowledge the limited sensitivity to detect true associations of being male with the selected variables.

Additionally, some other confounding factors might be associated with changes in dietary intake. For instance, we included the geographic residence of participants by county level, yet other environmental- and neighbourhoodlevel factors relating to food access, such as the availability of transportation and stores with healthy food selection ${ }^{(32)}$, were not included in the present study. We also chose a proxy measure of socioeconomic status, participants' educational levels $v$. using income levels. This decision was made based on the fact that a large proportion of missing values in income levels (19.4\%) were identified along with a moderate level of correlation between educational and income levels $(r 0.45$, $P<0.001$ ). We decided to use participants' education levels as a proxy measure of income levels in the current study because we did not have other wealth-related information available for participants.

\section{Implications}

The current study suggests valuable practice and research implications. Participants of Texercise Select, which stresses engagement through discussions and activities for goalsetting and problem-solving, improved dietary-specific social support for healthy eating measured over the programme period. The improved dietary-specific social support was partially related to increased fruit/vegetable intake among participants over time. Thus, this groupbased lifestyle intervention, which can facilitate social support for developing various healthy eating skills, may be beneficial for improving dietary behaviours among middle-aged and older adults. While the current study specifically examined Texercise Select, it is plausible that other group-based interventions that facilitate the development of practical skills through engagement (e.g. disease self-management) for older adults may be capable of improving dietary-specific social support and enhancing intended programme outcomes. Future research should investigate additional social support strategies for healthy eating. Such efforts would be beneficial for identifying workshop activities towards enhancing social support for healthy eating.

\section{Conclusion}

Middle-aged and older adults who participated in Texercise Select improved their dietary intake of weekly fruit/ vegetables and daily water intake as well as the level of dietary-specific social support compared with the comparison group. The improved level of dietary-specific social support among programme participants was associated with improved weekly fruit/vegetable intake. No association was found between the improved level of dietaryspecific social support and an increase in daily water intake. Furthermore, the intervention effect on improved weekly fruit/vegetable intake was mediated by an improved level of dietary-specific social support among programme participants, suggesting that Texercise Select may have 
helped improve their fruit/vegetable intake by increasing the levels of dietary-specific social support. Living with someone and being female were also associated with an improved intake of fruit/vegetable intakes. Designing a lifestyle intervention to promote social support for developing healthy eating skills may be critical to improving food intake among middle-aged and older adults. This, in turn, can promote healthy aging and reduce the onset and progression of chronic diseases.

\section{Acknowledgements}

Acknowledgements: The authors recognise the Texas Health and Human Services Commission for supporting the creation and evaluation of Texercise Select, and the Texas A\&M School of Public Health for helping to standardise and implement Texercise Select. We thank the delivery sites, class facilitators and participants for their role in the study. Financial support: None. Conflict of interest: There are no conflicts of interest. Authorship: A.Y. conceived research questions. A.Y. wrote the draft with M.L.S. and M.G.O. SL handled data acquisition and management. A.Y. performed statistical analyses. S.L., S.D.T., M.L.S. and M.G.O. provided critical revisions and feedback. All authors approved the manuscript. Ethics of buman subject participation: The current study was conducted according to the guidelines laid down in the Declaration of Helsinki, and all procedures involving research study participants were approved by the Texas A\&M University Institutional Review Board. Written informed consent was obtained from all participants.

\section{References}

1. Benjamin EJ, Muntner P, Alonso A et al. (2019) Heart disease and stroke statistics: 2019 update: a report from the American Heart Association. Circulation 139, e56-e 528.

2. Hales C, Carroll M, Fryar C et al. (2017) Prevalence of Obesity Among Adults and Youth: United States, 2015-2016, vol. 288. Hyattsville, MD: National Center for Health Statistics.

3. Flegal KM, Kruszon-Moran D, Carroll MD et al. (2016) Trends in obesity among adults in the United States, 2005 to 2014. JAMA 315, 2284-2291.

4. Abdullah A, Peeters A, de Courten M et al. (2010) The magnitude of association between overweight and obesity and the risk of diabetes: a meta-analysis of prospective cohort studies. Diabetes Res Clin Pract 89, 309-319.

5. Shlisky J, Bloom DE, Beaudreault AR et al. (2017) Nutritional considerations for healthy aging and reduction in age-related chronic disease. Adv Nutr 8, 17-26.

6. Hung HC, Joshipura KJ, Jiang R et al. (2004) Fruit and vegetable intake and risk of major chronic disease. $J$ Natl Cancer Inst 96, 1577-1584.

7. Ellingsen I, Hjerkinn EM, Seljeflot I et al. (2008) Consumption of fruit and berries is inversely associated with carotid atherosclerosis in elderly men. Br J Nutr 99, 674-681.

8. van de Rest O, Berendsen AA, Haveman-Nies A et al. (2015) Dietary patterns, cognitive decline, and dementia: a systematic review. Adv Nutr 6, 154-168.
9. Milte CM \& McNaughton SA (2016) Dietary patterns and successful ageing: a systematic review. Eur J Nutr 55 , 423-450.

10. Kesse-Guyot E, Andreeva VA, Jeandel C et al. (2012) A healthy dietary pattern at midlife is associated with subsequent cognitive performance. J Nutr 142, 909-915.

11. Jannasch F, Kröger J \& Schulze MB (2017) Dietary patterns and type 2 diabetes: a systematic literature review and meta-analysis of prospective studies. J Nutr 147, 1174-1182.

12. Qian F, Liu G, Hu FB et al. (2019) Association between plant-based dietary patterns and risk of type 2 diabetes: a systematic review and meta-analysis. JAMA Intern Med 179, 1335-1344.

13. Schwingshackl L, Missbach B, König J et al. (2015) Adherence to a Mediterranean diet and risk of diabetes: a systematic review and meta-analysis. Public Health Nutr 18, 1292-1299.

14. Lai JS, Hiles S, Bisquera A et al. (2013) A systematic review and meta-analysis of dietary patterns and depression in community-dwelling adults. Am J Clin Nutr 99, 181-197.

15. Govindaraju T, Sahle BW, McCaffrey TA et al. (2018) Dietary patterns and quality of life in older adults: a systematic review. Nutrients 10, 971.

16. Nicklett EJ \& Kadell AR (2013) Fruit and vegetable intake among older adults: a scoping review. Maturitas 75, 305-312.

17. Lee-Kwan S, Moore L, Blanck H et al. (2017) Disparities in statespecific adult fruit and vegetable consumption: United States, 2015. MMWR Morb Mortal Wkly Rep 66, 1241-1247.

18. Locher JL, Ritchie CS, Roth DL et al. (2005) Social isolation, support, and capital and nutritional risk in an older sample: ethnic and gender differences. Soc Sci Med 60, 747-761.

19. Boulos C, Salameh P \& Barberger-Gateau P (2017) Social isolation and risk for malnutrition among older people. Geriatr Gerontol Int 17, 286-294.

20. Sahyoun NR, Zhang XL \& Serdula MK (2006) Barriers to the consumption of fruits and vegetables among older adults. J Nutr Elder 24, 5-21.

21. Conklin AI, Forouhi NG, Surtees P et al. (2014) Social relationships and healthful dietary behaviour: evidence from over-50s in the EPIC cohort, UK. Soc Sci Med 100, 167-175.

22. McIntosh WA, Shifflett PA \& Picou JS (1989) Social support, stressful events, strain, dietary intake, and the elderly. Med Care 27, 140-153.

23. Vesnaver E \& Keller HH (2011) Social influences and eating behavior in later life: a review. J Nutr Gerontol Geriatr 30, $2-23$.

24. Bloom I, Edwards M, Jameson KA et al. (2016) Influences on diet quality in older age: the importance of social factors. Age Ageing 46, 277-283.

25. Davis MA, Murphy SP, Neuhaus JM et al. (2000) Living arrangements affect dietary quality for U.S. adults aged 50 years and older: NHANES III 1988-1994. J Nutr 130, 2256-2264.

26. Bloom I, Lawrence W, Barker M et al. (2017) What influences diet quality in older people? A qualitative study among community-dwelling older adults from the Hertfordshire Cohort Study, UK. Public Health Nutr 20, 2685-2693.

27. Stansfeld S \& Marmot M (1992) Deriving a survey measure of social support: the reliability and validity of the Close Persons Questionnaire. Soc Sci Med 35, 1027-1035.

28. Ory MG, Smith ML, Howell D et al. (2015) The conversion of a practice-based lifestyle enhancement program into a formalized, testable program: from Texercise Classic to Texercise Select. Front Public Health 2, 291-291.

29. Smith ML, Ory MG, Jiang L et al. (2015) Texercise Select effectiveness: an examination of physical activity and nutrition outcomes. Transl Behav Med 5, 433-442.

30. Smith ML, Lee S, Towne SD, et al. (2019) Impact of a behavioral intervention on diet, eating patterns, self-efficacy, and social support. J Nutr Educ Behav 52, 180-186. 
31. Ory MG, Lee S, Han G et al. (2018) Effectiveness of a lifestyle intervention on social support, self-efficacy, and physical activity among older adults: evaluation of Texercise Select. Int J Environ Res Public Health 15, E234.

32. Sharkey JR, Johnson CM \& Dean WR (2010) Food access and perceptions of the community and household food environment as correlates of fruit and vegetable intake among rural seniors. BMC Geriatr 10, 32.

33. Ingram DD \& Franco SJ (2014) 2013 NCHS urban-rural classification scheme for counties. Vital Health Stat 2, 166.

34. Kaiser HF (1974) An index of factorial simplicity. Psychometrika 39, 31-36.

35. Fornell C \& Larcker DF (1981) Evaluating structural equation models with unobservable variables and measurement error. J Mark Res 18, 39-50.

36. Plotnikoff RC, Lubans DR, Penfold CM et al. (2014) Testing mediator variables in a physical activity intervention for women with type 2 diabetes. Psychol Sport Exerc 15, 1-8.

37. Baron RM \& Kenny DA (1986) The moderator-mediator variable distinction in social psychological research: conceptual, strategic, and statistical considerations.J Pers Soc Psychol 51, 1173-1182.

38. Shrout PE \& Bolger N (2002) Mediation in experimental and nonexperimental studies: new procedures and recommendations. Psychol Methods 7, 422-445.

39. Field A (2013) Exploratory factor analysis. In Discovering Statistics Using IBM SPSS Statistics. Thousand Oaks, CA: Sage Publications Ltd.
40. Davis MA, Murphy SP \& Neuhaus JM (1988) Living arrangements and eating behaviors of older adults in the United States. J Gerontol 43, S96-S98.

41. Dean WR \& Sharkey JR (2011) Rural and urban differences in the associations between characteristics of the community food environment and fruit and vegetable intake. $J$ Nutr Educ Behav $\mathbf{4 3}$ 426-433.

42. Shanks C, Haack S, Tarabochia D et al. (2017) Factors influencing food choices among older adults in the rural western USA. J Community Health 42, 511-521.

43. Johnson AE, Donkin AJ, Morgan K et al. (1998) Fruit and vegetable consumption in later life. Age Ageing 27, $723-728$.

44. Nicklett EJ, Semba RD, Simonsick EM et al. (2012) Diet quality and social support: factors associated with serum carotenoid concentrations among older disabled women (the Women's Health and Aging Study). J Nutr Health Aging 16, 511-518.

45. Meenan RF, Mason JH, Anderson JJ et al. (1992) AIMS2: the content and properties of a revised and expanded arthritis impact measurement scales health status questionnaire. Arthritis Rheum 35, 1-10.

46. Glasgow RE (2013) What does it mean to be pragmatic? Pragmatic methods, measures, and models to facilitate research translation. Health Educ Behav 40, 257-265.

47. Althubaiti A (2016) Information bias in health research: definition, pitfalls, and adjustment methods. $J$ Multidiscip Healthc 9, 211-217. 